\title{
Abubillas, cucos y aves rapaces: la autoría de Sófocles, Fr. 581 R. (= Arist., HA 633a 17-28) (Tereo)
}

\author{
Miryam Librán Moreno \\ Universidad de Extremadura \\ mlibmor@unex.es
}
Hoopoes, cuckoos and birds of prey: the authorship of Sophocles, Fr. 581 R. (Arist., HA 633a 17-28) (Tereus)

\begin{abstract}
Algunos detalles mitográficos y ornitológicos de S., Fr. 581 R. (la transformación de Tereo en abubilla y gavilán; la conversión de Itis en ave rapaz), conservado en Aristóteles, HA 633a 17-28, son incompatibles con lo que se puede conocer de Tereo de Sófocles, lo que suscita dudas sobre la autoría del fragmento.
\end{abstract}

Palabras clave: Tereo; Itis; Sófocles; ornitología; Filocles; Aristóteles; Historia de los animales.
Some mythographical and ornithological details in S., Fr. 581 R. (Tereus' metamorphosis into both a hoopoe and a hawk; Itys' transformation into a raptor), preserved by Aristotle, $H A$ 633a17-28, are not compatible with what can be known of Sophocles' Tereus. Such a discrepance casts doubts on the Sophoclean authorship of the fragment.

Key words: Tereus; Itys; Sophocles; ornithology; Philocles; Aristotle; History of Animals.

\section{El PROBLEMA ${ }^{1}$}

Aristóteles (HA 633a17-28) transmite un fragmento trágico de diez versos que atribuye a Esquilo (A., Fr. 297 N. = S., Fr. 581 R.), en el que alguien revela el futuro que espera a Tereo tras la muerte de su hijo Itis a manos de su esposa, Procne, y la hermana de esta, Filomela ${ }^{2}$. Aristóteles aduce el fragmento como prueba de que la abubilla cambia de color y de aspecto a

${ }^{1}$ La autora desea manifestar su agradecimiento a los referees anónimos de Emerita, cuyas críticas y sugerencias han mejorado sustancialmente el presente artículo.

${ }^{2}$ Se puede consultar las fuentes literarias sobre el mito e.g. en Fontenrose 1948, p. 130 s., Sommerstein, Fitzpatrick y Talboy 2006, pp. 141-149, Milo 2008, pp. 125-134. 


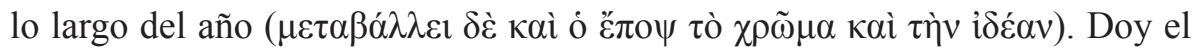
texto de Diggle $(1998)^{3}$ :

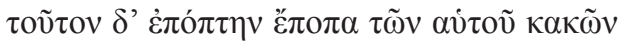

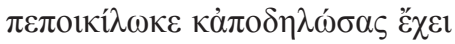

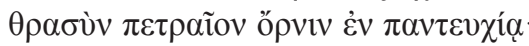

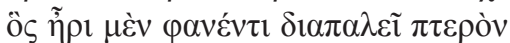

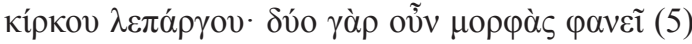

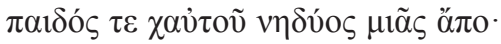

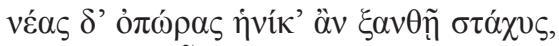

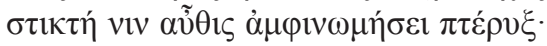

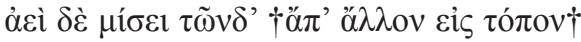

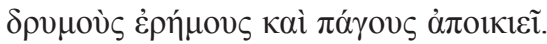

En cambio, a la abubilla de la que acabo de hablar, testigo de sus propios males, la ha pintado con colores variopintos, y la ha mostrado como ave audaz, habitante de las rocas, con toda la armadura puesta. Esta, cuando se presente la primavera, agitará alas de blanco gavilán, puesto que dos serán las formas que mostrará, la de su hijo y la de sí misma, al venir de un solo vientre. Pero a finales del verano, cuando se trille la espiga, alas moteadas lo rodearán de nuevo. Y siempre por odio $\uparrow$ a ellas irá a otro lugar $\uparrow$ a colonizar bosques solitarios y colinas.

Desde Welcker 1839, p. 384, la mayoría de estudiosos ha considerado que Aristóteles cometió un error de cita, dado que no hay constancia alguna de que Esquilo, aunque conocía bien el mito ${ }^{4}$, escribiera una tragedia sobre la materia de Tereo. Además, algunos rasgos lingüísticos aconsejan desechar la autoría esquilea, entre los que cabe señalar la perífrasis de $̌ x \omega \omega$ con participio de ao-

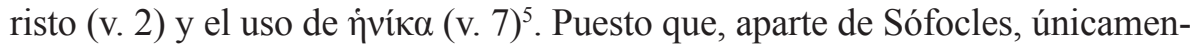
te Filocles (24 T Sn.-K.) entre los tragediógrafos griegos y Livio Andronico (frs. 24-9 W.) y Accio (frs. 639-66 W.) entre los latinos compusieron tragedias sobre este asunto, todos los editores modernos prefieren atribuir el fragmento

3 Hay muchos problemas textuales en este fragmento, y la traducción del v. 9 no es segura. Véase e.g. Radt $1999^{2}$, p. 437 s.

4 Véase Supp. 57-67, Ag. 1140-1149, Fr. 291 R. J. A. Hartung ap. Wecklein 1893, p. 615 pensaba que este fragmento procedía de Cretenses de Esquilo.

5 Welcker 1839, p. 384, Oder 1888, p. 543, Pearson 1917, p. 223 s., Sommerstein, Fitzpatrick y Talboy 2006, p. 189 s., Monella 2005, p. 93. 
transmitido por Aristóteles a Tereo de Sófocles ${ }^{6}$. Han surgido algunas voces que dudan de la autoría sofoclea ${ }^{7}$, pero no han aportado argumentos sustanciales para sustentar tal opinión, más allá de criterios subjetivos de estilo y gusto ${ }^{8}$. El propósito del presente artículo es analizar algunos detalles centrados en la ornitología mítica presentes en S. Fr. 581 R. que parecen estar en desacuerdo con lo que se puede conocer de Tereo de Sófocles (que no es tanto como para reconstruir la obra punto por punto y sin duda alguna, aunque sí suficiente para permitir algunas deducciones razonables), y comprobar si pueden ofrecer algún dato objetivo sobre la autoría del fragmento.

Como se sabe, Tereo de Sófocles estableció la forma más influyente del mito de Tereo y Procne ${ }^{9}$, que, con variaciones de detalle, conocemos gracias a Apollod. III 14.8, Ou., Met. VI 424-674 e Hyg., Fab. 45. Una opinión muy divulgada sostiene que Tereo de Sófocles introdujo dos innovaciones con respecto a las versiones anteriores, llamadas a conocer un inmenso éxito posterior (si bien siempre ha de tenerse en cuenta la flexibilidad, libertad y creatividad que están presentes en toda re-elaboración literaria de un mito): la ambientación del drama en Tracia, no en Dáulide, y la transformación de Tereo en abubilla, no en ave rapaz ${ }^{10}$. Ahora bien, en S. Fr. 581 R. hay dos detalles mitográficos que parecen incompatibles con lo que podemos deducir de Tereo de Sófocles: la transformación de Tereo tanto en abubilla como en gavilán (кíркоৎ) ${ }^{11}$ y la metamorfosis de Itis en ave rapaz.

${ }^{6}$ Lo incluyen en Tereo de Sófocles Pearson 1917, Radt 19992, Lloyd-Jones 1996, Diggle 1998, Sommerstein, Fitzpatrick y Talboy 2006, Milo 2008. Las excepciones modernas más notables son Cazzaniga 1950, pp. 37-41 y Mette 1959, p. 219, 1963, p. 196 n.1, que lo atribuyen a Esquilo ( $F r$. 609a M.). Nauck $1889^{2}$, p. 95 y Wecklein 1893, p. 614 s. (Fr. 304) todavía lo consideraban esquileo.

${ }^{7}$ Van Leeuwen 1902, p. 263 s. Algunos autores han propuesto que el autor puede ser Filocles (24 F 1 Sn.-K.), contemporáneo de Eurípides (24 T 1 Sn.-K.), autor de una tetralogía titulada Pandiónida, una de cuyas tragedias se denominaba Tereo o la abubilla (24 T 6c Sn.-K.). Véase Van Leeuwen 1902, p. 264 n. 4, Burnett 1998, p. 183 n. 22, Fitzpatrick 2001, p. 99 n. 58, March 2003, p. 161 n. 55. Filocles era sobrino de Esquilo, y pertenecía por tanto a la fecunda dinastía trágica establecida por el tragediógrafo de Eleusis (24 T 2 Sn.-K.).

${ }^{8}$ Cf. Allan 2004, Coo 2013, p. 378 n. 62.

9 Fontenrose 1948, p. 150, Cazzaniga 1950, p. 45, Forbes Irving 1990, p. 248, Monella 2005 , p. 83.

${ }_{10}$ Dobrov 1993, pp. 198-200, 212, Martín Rodríguez 2002, p. 67, Sommerstein, Fitzpatrick y Talboy 2006, p. 145, Scattolin 2013, pp. 131, 134.

${ }^{11}$ Utilizo el término general «gavilán», sin precisar especie, porque no está claro en qué tipo de ave rapaz paleártica se transforma Tereo: puede tratarse del gavilán común (accipiter nisus) (Dunbar 1995, p. 141), el fantasmal aguilucho papialbo (circus macrourus) (Arnott 2007, p. 99) o, en mi opinión, el gavilán griego (accipiter brevipes). 


\section{LA TRANSFORMACiÓN DE TEREO TANTO EN ABUBILla COMO EN AVE RAPAZ}

La metamorfosis más antigua de Tereo hace de él un falconiforme (кíрко૬, $i \varepsilon ́ p \alpha \xi$, accipiter), eterno enemigo y perseguidor del ruiseñor ${ }^{12}$. Sin embargo, en S., Fr. 581 R. se afirma que la transformación de Tereo es doble. Tereo se convierte primero en abubilla (1-3). En primavera y verano se transforma

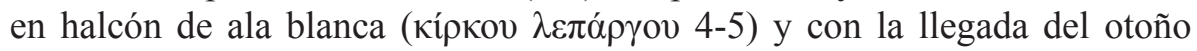
adquiere de nuevo la condición primera de abubilla (7-8).

Varios estudiosos han considerado que, en este fragmento, Sófocles pretendía establecer un puente entre la metamorfosis tradicional de Tereo en gavilán y la innovadora metamorfosis en abubilla introducida por él mismo, aprovechando una presunta creencia popular griega sobre la identidad de abubilla y gavilán común. Dos son los factores que, según numerosos autores, pudieron sugerir a la consciencia popular griega la semejanza entre abubilla y gavilán ${ }^{13}$. En primer lugar, destacan el supuesto parecido de la voz de la abubilla y el cuco, ave esta que efectivamente era confundida con el gavilán en Grecia (Plu., Arat. 30.8, cf. Arist., HA 563b14-29), y continúa siéndolo en el folclore europeo ${ }^{14}$. Según la reconstrucción del proceso de confusión entre cuco, abubilla y gavilán que hacen estos estudiosos, en el mito originario de Tereo, situado en Asia Menor, Tereo, transformado en cuco, perseguía al ruiseñor y a la golondrina preguntando siempre dónde estaban (кои̃ кои̃, «dónde, dónde»). Naturalmente, al migrar la leyenda de Jonia a Ática la voz

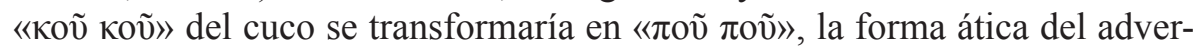
bio de lugar jonio koṽ. Dicho canto ya no se parece a la voz del cuco, sino

12 A., Supp. 60-61, Ach. Tat. V 3.3, D.P., Au. I 6, Hyg., Fab. 45, Demetr.Pepag., Hierakosophion 11. Véase Escalígero 1573, p. 415, Oder 1888, p. 547, Biraud-Delbey 2006, p. 6, Monella 2005, p. 120.

${ }^{13}$ Escalígero 1573, p. 415: «Fefellit Aeschylum, quod Epops eandem plane vocem cum Cucullo emittit. Propterea eundem Epopem cum cucullo fecit; qui, ut nemo ignorat, tam similis est accipitri, ut quidam in genere accipitrum posuerint», Welcker 1839, p. 385, Oder 1888, pp. 548-550, Thompson 1895, p. 55, Van Leeuwen, 1902, pp. 261, 262 n.5, Schroeder 1926, p. 434, Forbes Irving 1990, p. 249, Dobrov 1993, p. 212, Dunbar 1995, p. 141, March 2003, p. 47 n.22, Casanova 2006, p. 177, Biraud-Delbey, 2006, p. 5 s., Scattolin 2013, p. 131.

${ }^{14}$ Kunstmann 1938, p. 35, Arnott 2007, p. 101. El parecido entre el cuco y el gavilán responde a la realidad ornitológica, ya que la estrategia del cuco para minimizar la resistencia de sus anfitriones es tener un plumaje y forma de vuelo semejantes a los del gavilán, temible depredador de la mayoría de pajarillos cuyo nido parasita el cuco. Véase Welbergen y Davies 2011, pp. 574-579. 
al canto nupcial de la abubilla en primavera. Así se pasaría de una asimilación cuco $>$ gavilán a cuco $>$ abubilla $>$ gavilán, y por extensión a la doble metamorfosis de Tereo en abubilla y ave rapaz. En segundo lugar, el nombre

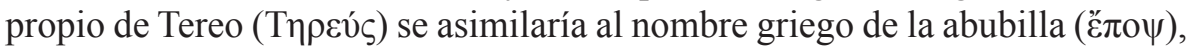
interpretados ambos en el sentido de 'el que observa, el que vigila' ( $\dot{\varepsilon} \pi \mathrm{o} \pi \tau \varepsilon v ́ \varepsilon i v$, Sch. Ar., Au. 102), lo que coincidiría con uno de los rasgos dis-

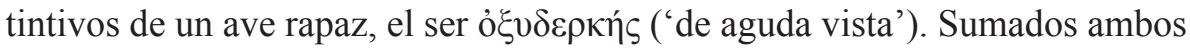
factores al desconocimiento que tenía la mayoría de griegos del aspecto de la abubilla $^{15}$, continúa el argumento, sería fácil explicar así la confusión entre abubilla y ave rapaz.

Sin embargo, esta explicación del paso de gavilán a abubilla por confusión de ambos con el cuco, pese a estar muy divulgada, no se sostiene. Dejemos de lado la imposibilidad total de confundir a la abubilla con ave rapaz alguna, tanto por las enormes diferencias de tamaño y comportamiento como de vuelo $^{16}$, detectables a simple vista y sin necesidad de mucha observación. Omitamos también que la abubilla reside en primavera y verano en Grecia, mientra que el gavilán común es invernante, por lo que la secuencia temporal descrita en S., Fr. 581 R. es errónea ${ }^{17}$. No se puede quizá exigir a los antiguos griegos, que carecían de los instrumentos de óptica actuales para la observación de las aves, un conocimiento tan minucioso del aspecto y la etología de un ave de campo abierto como la abubilla. Dejemos también de lado que muchos animales (mamíferos y reptiles no menos que aves) eran considera-

${ }_{15}$ Van Leeuwen 1902, p. 264, Oder 1888, pp. 544-546. La información se repite todavía e.g. en Dubel 2006, p. 40 n.8. Esta idea parte de Ar., $A u$. 102, pero la broma de Aristófanes no se basa en la ignorancia del aspecto de la abubilla, sino en el desconocimiento de la apariencia del exótico pavo real, criatura casi mitológica y emblema de sofisticación y lujo extremo. Véase Sommerstein 1991², p. 205 s., Dunbar 1995, p. 165.

${ }^{16}$ Biraud - Delbey 2006, p. 5. La abubilla tiene una envergadura de 42 a $46 \mathrm{cms}$, mientras que los candidatos a ser кíркоऽ $\lambda \dot{\varepsilon} \pi \alpha \rho \gamma о \varsigma$ son significativamente mayores: el gavilán tiene entre 58 y $80 \mathrm{cms}$. de envergadura (según su sexo), mientras que el aguilucho papialbo entre los 100 y $150 \mathrm{cms}$. Véase Hume 2011, pp. 106, 111, 227, 393. La forma de volar de la abubilla, además, es imposible de confundir con la de un ave rapaz, o incluso con la de otra ave europea (Hume 2011, p. 227): su vuelo es inestable y arrítmico, con cortas ondulaciones y brevísimos planeos (Svensson 2010, p. 238), muy parecido al de una mariposa gigante, mientras que el vuelo del gavilán es recto y sumamente veloz, con aleteos rápidos y profundos como una paloma, mezclados con planeos cortos (Svensson 2010, p. 112, Hume 2011, p. 106).

${ }_{17}$ Dunbar 1995, p. 141. 


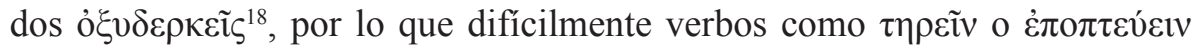
conducirían inmediatamente a pensar en un falcónido, en tanto en cuanto la

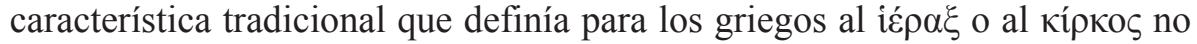
era su vista (a diferencia del águila), sino la gran velocidad de su vuelo y la sonoridad de su llamada ${ }^{19}$.

El argumento definitivo en contra de una presunta creencia folclórica sobre la identidad entre cuco y abubilla es doble: en primer lugar, el mito de Tereo llegó al Ática desde Mégara ${ }^{20}$, no desde Jonia, por lo que el paso

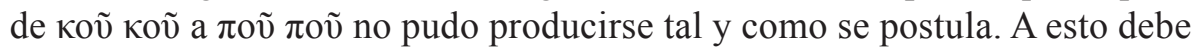
añadirse otro argumento igualmente contundente: no hay prueba alguna que asocie a Tereo con el cuco en ninguna de las muchas versiones documentadas del mito. En segundo lugar, no hay prueba alguna de que los griegos confundieran o asimilaran el canto del cuco y la abubilla (cosa que ocurre todavía a observadores poco avezados en Europa): los antiguos griegos sabían bien que el canto del cuco consistía en un sonoro ко́ккv (cf. Ar., $A u$. 505), mientras que el de la abubilla era un rápido estacato hueco, aflautado

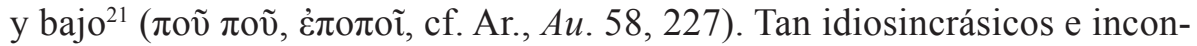
fundibles son los cantos del cuco y la abubilla que ambas aves recibieron

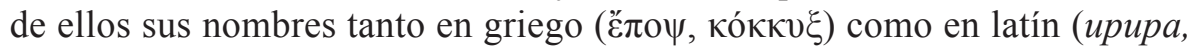

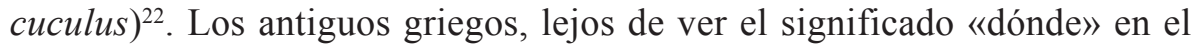
Ко́ккv del cuco, confundían el canto de este pájaro con el del gallo ${ }^{23}$, no con el de la abubilla, y además lo consideraban una señal de alarma (Ar., Ra.1380, 1384, Ec. 31). La diferencia clara que percibían los griegos entre las voces del cuco y la abubilla se refleja también en el verbo que describe

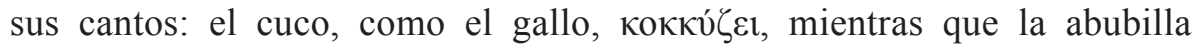

${ }_{18}$ Así son calificados el león (Man.Hist. 13, Et. Gud. $\lambda$ 367), la serpiente (Philox. Gramm. 471, Sch. Lyc. 673, Sch. Ar., Pl. 733), el ciervo (Eust.Ant., Hex. M.18.740), el águila (Eust., Il. 1122), el toro (Sch. Lyc. 553), el lince (Phot. $\lambda$ 432, Sud. $\lambda$ 777), la gacela (EM 286), el lobo (Sch. Arat. 747) y el halcón (Sch. Lyc. 148).

19 E.g. Eust., Il. 1262, 1300, con varios ejemplos de Homero.

${ }^{20}$ Hiller 1886, p. 48 s., Oder 1888, pp. 546, 553, Van Leeuwen 1902, p. 262 n. 5, Cazzaniga 1950, p. 28.

${ }_{21}$ Svensson 2010, p. 238, Hume 2011, p. 227.

${ }^{22}$ Keller 1913, pp. 60, 63, Chantraine $1999^{2}$, pp. 362, 554.

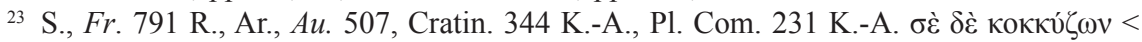
$>\dot{\alpha} \lambda \varepsilon \dot{\varepsilon} \kappa \tau \omega \rho \pi \rho о \kappa \alpha \lambda \varepsilon i \tau \tau \iota l$ («a ti te convoca el gallo gritando `co-co’»), Arist., HA 631b28, Eust., Od. 1479, y v. Dunbar 1995, p. 345, Arnott 2007, p. 10. 


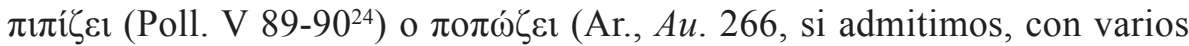
editores, la conjetura de Schroeder). A esto debe sumarse que tampoco es cierto que los antiguos griegos no estuvieran bien familiarizados con el aspecto y los hábitos de la abubilla: el público de Aristófanes estaba suficientemente informado sobre la apariencia, el comportamiento, el canto y la dieta de dicha ave, nidificante frecuente y residente primaveral y estival en el norte y centro de Grecia ${ }^{25}$. Esto es, si nos basamos en el testimonio de los propios griegos, y no en las observaciones de europeos actuales, no hay prueba alguna de que se identificara o relacionara el canto de cuco y abubilla en Grecia ${ }^{26}$.

Por tanto, la confusión entre abubilla y cuco por un lado y abubilla y gavilán por el otro no parece ser un dato mítico tradicional, y tampoco procede de una creencia popular o folclórica griega. Sólo podría cometerla alguien que no estuviera familiarizado en absoluto con la realidad ornitológica, cosa difícil de creer en una sociedad pre-industrial y agrícola que conocía suficientemente bien los hábitos de las aves que vivían en su vecindad ${ }^{27}$, o que decidiera torcerla a sabiendas para sus propósitos literarios. Es decir, se trataría de un intento individual aislado, no perteneciente a una tradición mítica o folclórica.

Si Sófocles fuera responsable de la idea de que la abubilla se transforma en gavilán en primavera, o si fuera esta una idea popular preexistente, indudablemente habría quedado algún otro rastro en la literatura griega (como han quedado huellas abundantes de la transformación del cuco en gavilán ${ }^{28}$ ), máxime habida cuenta de la inmensa popularidad de su Tereo y lo chocante que

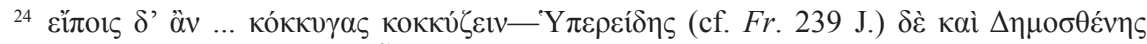

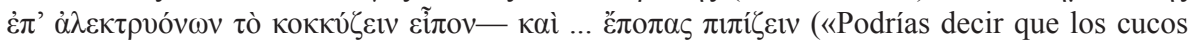
hacen 'cu-cu' - aunque Hipérides y Demóstenes atribuyeron el hacer 'cu-cu' a los gallos - y que ... las abubillas hacen 'pi-pi'»).

${ }_{25}$ Pollard 1977, pp. 14, 194, Dunbar 1995, p. 140, Arnott 2007, p. 45. La abubilla aparece por primera vez en Epich. 164 K.-A., en compañía de dos especies de aves perfectamente bien conocidas por los griegos, los autillos europeos ( $\sigma \kappa \tilde{\omega} \pi \alpha \varsigma)$ y los mochuelos europeos ( $\gamma \lambda \alpha \tilde{\kappa} \kappa \alpha \varsigma)$. La apariencia, dieta, hábitat y canto de la abubilla están correctamente descritos en Ar., $A u$. 32, 82, 94, 99, 279 y Arist., HA 488b2-3, 599a9-11, 615a15-16. Véase Keller 1913, pp. 61, 63.

${ }^{26}$ En el centro de Europa existe una creencia popular sobre la amistad entre la abubilla y el cuco (Oder 1888, p. 549 s., Kunstmann 1938, pp. 30, 38), cosa que pudo haber influido, posiblemente de forma subconsciente, en la creación de dicha teoría.

27 Pollard 1977, p. 13 s., Dunbar 1995, pp. 141, 227.

28 Aesop. 198, Plu., Arat. 30.8, cf. Arist., HA 563b14-29. 
sería tal doble metamorfosis ${ }^{29}$. Sin embargo, no parece haber en la literatura griega otro testimonio de que la abubilla se transforme en gavilán aparte del fragmento que nos ocupa: las únicas fuentes que mencionan dicha doble metamorfosis dependen directamente de la noticia de Aristóteles ${ }^{30}$. En realidad, ni siquiera es del todo exacto que Aristóteles siga al autor del fragmento en la idea de que la abubilla se convierte en gavilán. Aristóteles se limita a citar el pasaje como testimonio de su tesis (errónea) de que la abubilla tiene un color $\mathrm{y}$ aspecto distinto en invierno del que tiene en primavera, semejante al del кípкоs, no para abonar el cambio de una especie de ave a otra ${ }^{31}$.

Como se ha dicho, pese a que hay que tener siempre presente la libertad individual creativa de los escritores posteriores, lo cierto es que la tragedia de Sófocles estableció la forma más influyente del mito de Tereo y Procne ${ }^{32}$.

${ }^{29}$ Cazzaniga 1950, p. 38.

${ }^{30}$ Thphr., HP II 4.4, Plin., NH X 86 y Gp. I 22 dependen estrechamente de la noticia de Aristóteles, por lo que no pueden ser tomados como testigos independientes. Véase Van Leeuwen 1902, p. 264 n. 6.

${ }^{31}$ Así lo entendió Escalígero 1573, p. 415: «Deinde alium plumarum colorem illi vere

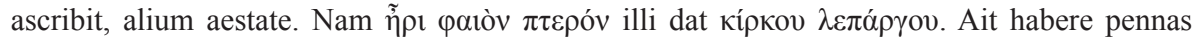
accipitris, qui est $\lambda \dot{\varepsilon} \pi \alpha \rho \gamma o \zeta$, hoc est ventro albo, et habet $\varphi \alpha$ òv $\pi \tau \dot{\varepsilon} \rho \circ v$, hoc est fuscas pennas». La idea de que Aristóteles atestigua la transformación de abubilla en gavilán procede de una interpretación errónea de la naturaleza muy heterogénea del capítulo 49B, en el que se narran los cambios estacionales de las aves (HA 632b-633a). Entre dichos cambios se cuentan el cese del canto primaveral ( $\varphi \omega v \eta \dot{)}$ del ruiseñor y la paloma torcaz ( $\dot{\eta} \delta$ ' $\dot{\eta} \eta \dot{\omega} v \ldots \dot{\eta}$ $\varphi \alpha ́ \tau \tau \alpha)$, el cambio de color $(\chi \rho \tilde{\omega} \mu \alpha)$ del plumaje del pájaro debido a la muda postnupcial de

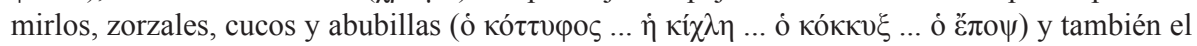
cambio de especie ( $\varepsilon \tilde{i} \delta \circ \varsigma$ ) de petirrojos y colirrojos reales, currucas cabecinegras y carboneros

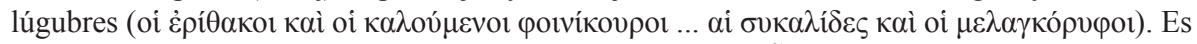
decir, el cambio estacional de la abubilla no es de especie ( $\varepsilon \tilde{i} \delta \circ \varsigma$ ), como ocurre con petirrojos y colirrojos reales por un lado, y currucas cabecinegras y carboneros lúgubres por el otro, sino de aspecto (i $\delta \delta \varepsilon \alpha)$ y color $(\chi \rho \tilde{\omega} \mu \alpha)$, como mirlos, zorzales, cucos y otros pájaros, debido

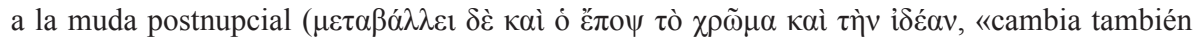

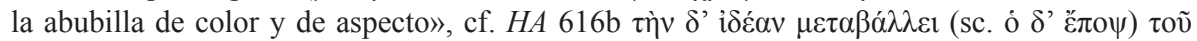

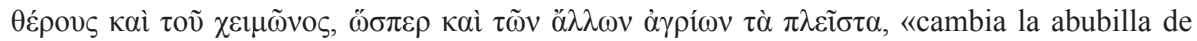
aspecto en invierno y en verano, como la mayoría de aves silvestres»). La creencia en una fuerte muda invernal de la abubilla que altera su aspecto aparece también en Ar., Au. 103-105:

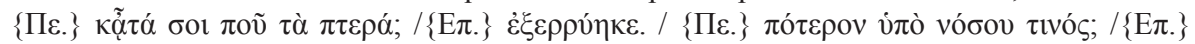

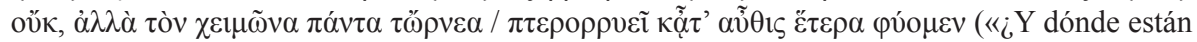
tus plumas? / Se me han caído. / ¿Por alguna enfermedad? / No, es que durante el invierno a todos los pájaros se nos caen las plumas y luego nos crecen otras»).

32 Véase supra n. 9. 
Incluso algunos estudiosos sostienen que, a partir del estreno de Tereo, la mayoría de alusiones al mito de Tereo que encontramos en la literatura grecolatina se refiere en último término a la tragedia de Sófocles ${ }^{33}$. Por tanto, si la metamorfosis alterna de Tereo en abubilla y posteriormente en gavilán se debiera a la celebérrima tragedia de Sófocles, sería esperable encontrar al menos alguna alusión a ella en las múltiples versiones literarias grecolatinas posteriores de dicho mito ${ }^{34}$. Sin embargo, ninguna de las fuentes literarias que dependen claramente de Tereo de Sófocles mencionan la transformación de Tereo en gavilán: en ellas únicamente se documenta la conversión en abubi1la ${ }^{35}$, y de hecho Ar., Au. 96-101 demuestra que la transformación física de Tereo en abubilla era uno de los detalles de Tereo de Sófocles más recordados por el público ${ }^{36}$. Como se ha mencionado anteriormente, se ha pensado que Sófocles pudo haber introducido la doble metamorfosis en ave rapaz y en abubilla para justificar la rompedora innovación que supone convertir a Tereo en abubilla, y servir así de transición desde la versión tradicional que hacía de Tereo un ave rapaz ${ }^{37}$. Con todo, no es cierto que fuera Sófocles el primero en introducir la transformación de Tereo en abubilla, pese a que es esta una opinión repetida con frecuencia ${ }^{38}$ : esta metamorfosis, lejos de ser invención de Sófocles, estaba ya bien divulgada en la versión megárica del mito (Paus. I 41.8-10), la más antigua de la Grecia continental, desde donde posteriormente viajó a Atenas ${ }^{39}$.

33 Dobrov 1993, p. 212, Brown 2002, p. 219, Monella 2005, p. 83.

${ }^{34} \mathrm{La}$ comparación de Tereo con un águila y con otra ave rapaz inespecífica en Ou. Met., VI 516-517, 530 es un símil de raíz épica (Friis Johansen y Whittle 1980, p. 179, Martín Rodríguez 2002, p. 163 s.) mediante el cual Ovidio demuestra juguetonamente su consciencia de la existencia de la otra versión (Kaufhold 1997, p. 68 n. 12), sin presentarlas concurrentemente.

${ }^{35}$ Cazzaniga 1950, p. 59. De hecho, la principal fuente (exceptuando a Esquilo) que hace de Tereo un gavilán, la fábula 45 de Higino, se separa significativamente en muchos aspectos de Sófocles. Véase e.g. Monella 2005, p. 129, Milo 2008, p. 127.

${ }^{36}$ Véase e.g. Wright 2012, p. 589.

37 Cf. Dobrov 1993, pp. 212, 218.

38 Todavía aparece e.g. en Hanhemann 2012, p. 175, Scattolin 2013, p. 131.

${ }^{39}$ Hiller 1886, p. 48 s., Oder 1888, pp. 546, 553, Van Leeuwen 1902, p. 262 n. 5, Cazzaniga 1950, p. 28, Martín Rodríguez 2002, p. 42. Los fragmentos de una hidria del Pintor de Altamura (ARV ${ }^{2}$ 594.55), datable entre $470-460$ a. C. y por tanto sustancialmente anterior a Tereo de Sófocles, parecen atestiguar ya la transformación de Tereo en abubilla. Véase March 2000, p. 129 n. 28. 
Así pues, la metamorfosis de Tereo en abubilla ya figuraba en versiones del mito anteriores a Sófocles, por lo que sería innecesario que el tragediógrafo explicara con tanto detalle una presunta innovación, que no es tal y que desde luego no era suya, con respecto a la transformación tradicional de Tereo en gavilán. Más verosímil sería que, posteriormente, alguien tratara de conciliar la conversión en кípкоৎ que atestigua Esquilo (Supp. 5767) con la célebre transformación en abubilla de Tereo de Sófocles y que ese alguien fuera mucho menos conocido literariamente que Sófocles, hasta el punto de resultar de interés científico para Aristóteles, pero no literario.

\section{LA METAMORFOSIS DE ItIS EN AVE RAPAZ}

Hay en el $F r$. 581 una expresión de significado muy oscuro y discutido ${ }^{40}$, que quizá pueda desvelar la razón de la extraña doble metamorfosis de Tereo en abubilla y gavilán que se documenta en este fragmento y revele un nuevo detalle incompatible con Sófocles. Heath 1762, p. 164 entendió que los críp-

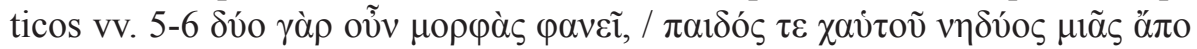
(«pues revelará dos formas, la de su hijo y la suya propia de un solo vientre», «for he will reveal two forms from a single womb, his son's and his own», trad. de L. Coo) hacen referencia al aspecto diferente del juvenil de abubilla con respecto al ave adulta ${ }^{41}$. Sin embargo, el inmaduro de abubilla no es significativamente distinto del adulto, a diferencia de lo que ocurre con otras aves. Es necesario buscar otra explicación.

En realidad, la introducción de las partículas yó $\rho$ oũv en el v. 5 explica la razón necesaria y esencial ${ }^{42}$ por la que Tereo, inicialmente convertido en abubilla (vv. 1-2), se transforma en gavilán cuando llega la primavera (ôs ท̃

${ }^{40}$ Cf. Pearson 1917, p. 227: «The expression is so obscure as to be scarcely intelligible», Pollard 1977, p. 165.

${ }^{41}$ «For he will display two forms coming from a single birth, the fledgeling's and his own» (Pearson 1917), «for he will display two shapes, that of his chick as well as his own, born from one belly» (Sutton 1984, p. 129). Otros han creído que la confusión se debe a un supuesto parecido entre la abubilla inmadura y el gavilán (así Forbes Irving 1990, p. 248 s.), aunque en realidad tal parecido no existe, como la consulta de cualquier guía ornitológica puede demostrar.

${ }^{42}$ Denniston $1954^{2}$, p. 446 : «in post-Homeric Greek oṽv adds to yó $\rho$ the idea of importance or essentiality». 


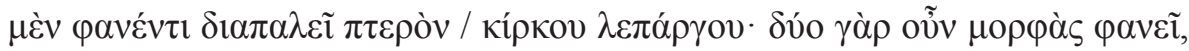
vv. 4-5). Puesto que ha devorado a Itis en un acto de canibalismo involunta-

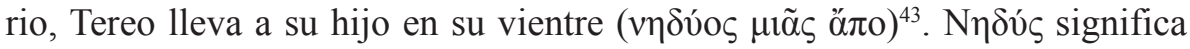
tanto «estómago» como «útero», y en el v. 5 están presentes ambos signifi$\operatorname{cados}^{44}$. Dado que Tereo tiene en su vientre ( $\left.v \eta \delta \dot{s} \varsigma\right)$ el cuerpo de su hijo, la

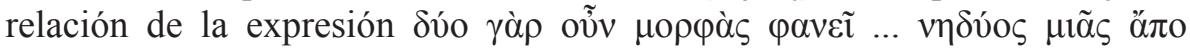
(«traerá a la luz dos formas de un solo vientre») con un parto simbólico parece evidente ${ }^{45}$. Cuando llega la primavera,Tereo/abubilla se convierte en joven gavilán (v. 4), la forma aviar que adopta su hijo (v. 5), el cual ha salido del propio vientre de su padre (v. 5) en inversión macabra de su ingesta ${ }^{46} \mathrm{y}$ en parodia perversa del proceso de gestación y alumbramiento ${ }^{47}$. Los vv. 5-6 sugieren que Tereo/abubilla, antropófago en su forma humana, debe transformarse también en кípкоร, ave notoriamente «caníbal» por su alimentación

${ }^{43}$ Cf. Sommerstein, Fitzpatrick y Talboy 2006, p. 192: «there must surely be an allusion to Tereus' act of cannibalism, whereby he took his son into his own belly», Milo 2008, p. 102: «il duplice aspetto dell' upupa-Tereo ... è verisimilmente da intendersi come quello suo proprio e come quello del figlio che egli ha divorato».

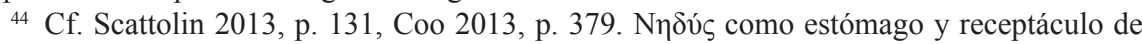
comida y bebida aparece en E., Cyc. 244, 303, 574, Fr. 282.5 K., S., OC 1263, Fr. 457 R. Con el significado de 'útero' aparece e.g. en Il. XXIV 496, Hes., Th.460, A., Eu. 665, E., Fr. $107.1 \mathrm{~K}$.

${ }^{45}$ Cf. Martín Rodríguez 2002, p. 254.

${ }^{46}$ Cf. Coo 2013, p. 379: «The metamorphosis of Tereus-hoopoe into Tereus-hawk is thus envisaged as a transformation into his son's avian form, which has emerged, as it were, from his own belly .... Working with this model, the emergence of the hawk-form from Tereus's stomach can be envisaged as a vomiting-up, a macabre reversal of Itys's ingestion». Cf. Ou., Met. VI 663-664 et modo, si posset, reserato pectore diras / egere inde dapes semesaque viscera gestit («y ya, si pudiera, desea abrir su pecho y echar fuera las terribles viandas y la carne de su carne», trad. de A. Ma Martín Rodríguez).

${ }^{47}$ La misma mezcla de deglución y gestación se documenta en Ou., Met. VI 651 inque suam sua viscera congerit alvum, «(Tereo) engulle y concentra en su vientre sus propias entrañas» (trad. de A. Ma Martín Rodríguez) y es un rasgo fundamental del relato ovidiano del mito de Tereo. Véase Martín Rodríguez 2002, pp. 202, 246, 254, Gildenhard - Zissos 2007, p. 10, Coo 2013, p. 379 s.: «Tereus simultaneously stuffs meat into his stomach and .... fills up his 'womb' with his own child. The idea is repeated later, when Tereus longs in vain to be able to open up his own breast in order to remove dapes semesaque uiscera .... from within himself, again an image that depicts Itys as an object of both digestion and impossible parturition. In both Sophocles and Ovid the language slips between the different meanings to activate an image of Tereus's child-eating as a macabre act of impregnation». 
exclusivamente ornitófaga ${ }^{48}$, y ello es así precisamente por haber engullido, vomitado y «alumbrado» a otro pájaro, su propio hijo, cuya forma ha de adoptar. Por tanto, los vv. 5-6 parecen implicar que Itis, tras su muerte, fue transformado en algún tipo de ave, verosímilmente un ave rapaz.

Que Itis sea transformado en ave rapaz después de su muerte puede sorprender, indudablemente. Sin embargo, el nombre 'Itus se asocia etimológicamente con ítv $\xi^{49}$, denominación de un ave que posiblemente sea el milano real o el milano negro (Phot. 1 268; Sud. 1754$)^{50}$. Con todo, es difícil creer que dicha metamorfosis de Itis en ave rapaz figurara en la celebérrima tragedia de Sófocles, toda vez que su eco en la literatura posterior es absolutamente nulo. Además, tal transformación compite con variantes atestiguadas en otros autores, según los cuales Itis, tras ser desmembrado y devorado, fue transformado en fassa (Seru., Buc. VI 78) ${ }^{51}$, paloma torcaz, o en phasianus, faisán común (Myth. Vat. I 4). La existencia de grandes divergencias en cuanto al destino final de Itis parece sugerir que, en este caso concreto, no existía una fuente literaria de suficiente peso específico como para imponerse a versiones concurrentes y hacer de la metamorfosis de Itis en ave rapaz un elemento mayoritario del mito, cosa que sí había ocurrido con la transformación de Tereo en abubilla (en lugar de ave rapaz o pico

${ }^{48}$ El кíркоৎ tenía fama tradicional como devorador inmisericorde de otras aves (Friis Johansen y Whittle 1980, p. 179). Una fusión comparable entre varón que traspasa los límites morales y pretende cometer incesto (Sandin 2005, p. 140) у кíрко caníbal que devora a sus congéneres ( $\gamma \varepsilon \dot{v}(\varsigma)$ ), las aves, aparece en A., Supp. 223-228, precisamente en el contexto de

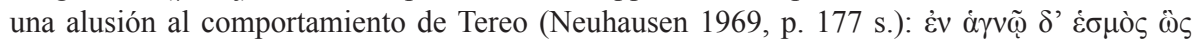

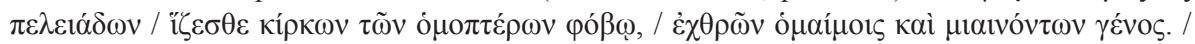

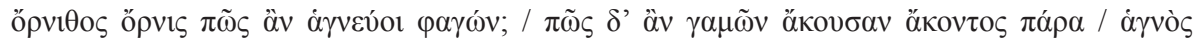

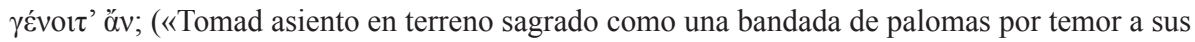
congéneres los gavilanes, enemigos de sus consanguíneos y mancilladores de su propia raza. ¿Cómo podría ser puro un pájaro que ha devorado a otro pájaro? ¿Y cómo uno que desposa a una mujer contra su voluntad y la de su padre podría ser puro?»). Véase Friis Johansen y Whittle 1980, pp. 179-182, Sandin 2005, p. 139 s.

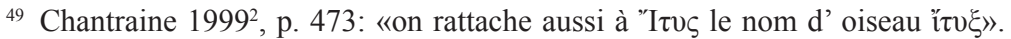

${ }^{50} \mathrm{El}$ ave denominada $\iota^{\prime} v \xi \xi$ es verosímilmente idéntica a $₹ \delta v \xi$, uno de los nombres del mi-

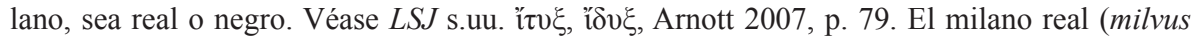
milvus) y el milano negro (milvus migrans), evidentemente, no pertenecen al mismo género que el gavilán común (accipiter nisus), pero los antiguos griegos tendían a asociarlos con los

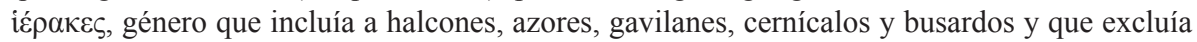
a águilas, buitres y rapaces nocturnas. Véase Aubert-Wimmer 1868, p. 92.

${ }^{51}$ Fassa aparece también como glosa interlineal a Itys en tres MS de Hor. Carm. IV 12 (Peerlkamp 1834, p. 424). 
picapinos), cuya prevalencia se debe fundamentalmente a la gran influencia de Tereo de Sófocles ${ }^{52}$. Por otra parte, la supuesta conversión de Itis en ave es consistente con el hecho de que, en versiones post-sofocleas, las transformaciones en pájaros afectan a más miembros de la familia, y no sólo a Tereo, Procne y Filomela. La Ornitogonía de Beo (Ant. Lib., Met. XI 10) atestigua que Pandáreo, padre de Aedón (= Procne), se transforma en águila pescadora, su esposa en martín pescador, el hermano de Aedón en abubilla y Politecno (= Tereo) en pico picapinos. Ps. Acro $(C$. IV 12.6) transmite una versión, que podría remontarse a Livio Andronico (cf. $F r .27$ W. $)^{53}$, en la cual hasta la nodriza de Itis, llamada Daulíade, es transformada en paloma torcaz (in palumbem versa est).

En suma, la doble metamorfosis de Tereo en ave rapaz y abubilla (vv. 1-6) y la transformación de Itis en ave rapaz parece apuntar a una fuente que no se ajusta a lo que podemos deducir de Tereo de Sófocles.

Con respecto a la autoría de S., Fr. 581 R., con los datos actuales es imposible demostrar quién compuso dicho fragmento, y lo único que cabe aseverar con alguna certeza es que no parece proceder de Tereo de Sófocles. Algunos estudiosos han propuesto que el fragmento debe atribuirse a la tetralogía Pandiónida de Filocles, una de cuyas tragedias se titulaba Tereo o la abubilla (24 T 6c Sn.-K.) ${ }^{54}$. Es cierto que, fuera de Sófocles, únicamente Filocles entre los tragediógrafos griegos compuso una tragedia sobre el asunto de Tereo, Procne y Filomela ${ }^{55}$. Tereo de Filocles debió de ser algo parecido a un succès de scandale en su época, si tenemos en cuenta que Aristófanes consideró esta tragedia suficientemente importante como para burlarse de ella en Aves (280-281). El hecho de que tanto la poesía de Filocles como su aspecto poco agraciado sirvieran de chanza frecuente a los comediógrafos ${ }^{56}$ indica que su obra debió de ser bastante conocida en su época, como no podía ser de otra forma en el caso de un autor que era el mismísimo sobrino de Esquilo, tenía algo de la grandeza de su glorioso tío (cf. Telecl. $15 \mathrm{~K} .-\mathrm{A} .=24 \mathrm{~T} 8 \mathrm{~b} \mathrm{Sn} . \mathrm{K}$.), era padre de los conocidos tragediógrafos Mórsimo y Melantio (24 T 2 Sn.-K.), y había logrado la increíble hazaña de derrotar al Edipo Rey de Sófocles en el concurso trágico (24 T3a Sn.-K.). Por ello, es muy probable que su tetralogía más famosa llega-

52 Véase supra n. 10.

53 Privitera 2007, p. 30.

54 Véase supra n. 7.

55 Radke 1957, p. 247 s., Radt 1999², p. 437.

56 Cf. e.g. Ar., V. 461-462, Au. 280-281, 1295, Th. 168, Fr. 591.43-6 K.-A., Cratin. 323 K.-A. 


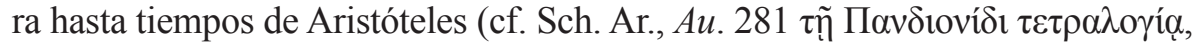

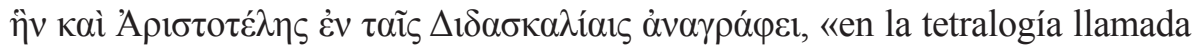
Pandiónida, de cuya existencia Aristóteles deja constancia también en sus $D i$ dascalias»). Aristóteles tendría plena consciencia de que el fragmento que aducía en $H A$ 633a 17-28 como prueba de los cambios estacionales de la abubilla no podía pertenecer a Sófocles debido a las diferencias de detalle con Tereo de dicho autor. Es verosímil que por ello atribuyera a Esquilo un pasaje compuesto por un sobrino del gran tragediógrafo ${ }^{57}$, que incluía (parcialmente) la metamorfosis de Tereo en кípкоৎ seguida por Esquilo (Supp. 62) y que difería significativamente de Tereo de Sófocles $^{58}$. Por desgracia, la ausencia de datos incontrovertibles sobre el contenido de la intrigante tragedia de Filocles impide que la atribución de nuestro fragmento a su Pandiónida no sea más que una hipótesis, atractiva pero en último término indemostrable.

\section{Conclusiones}

Dos detalles mitográficos y ornitológicos de S., Fr. 581 R. (la transformación de Tereo en abubilla y gavilán; la conversión de Itis en ave rapaz) son incompatibles con lo que se conoce del Tereo de Sófocles y debería valorarse si procede su eliminación de futuras ediciones y comentarios de dicha obra. El fragmento podría pertenecer a Filocles, aunque en último término no cabe pronunciarse sobre un autor concreto.

\section{BibLIOGRAFÍA}

Allan, W. 2002: Reseña de A. H. Sommerstein (ed.), Shards from Kolonos: Studies in Sophoclean Fragments, BMCR 2004.12.30 <http://bmcr.brynmawr. edu/2004/2004-12-30.html> (04/03/2014).

${ }^{57}$ Cf. Fitzpatrick 2001, p. 99 n. 58, Sommerstein - Fitzpatrick - Talboy 2006, p. 190, Hanhemann 2012, p. 175.

${ }^{58}$ La autoría de Filocles, sobrino de Esquilo y rival de Sófocles, podría explicar la mezcla de rasgos de dicción y estilo esquileos (sobre los cuales véase Cazzaniga 1950, p. 40s.), que se superponen a usos estrictamente sofocleos como la perífrasis de Ě $\chi \omega$ más participio de aoristo. Aristófanes $(A u$. 280-281) parece acusar veladamente a Filocles de plagiar Tereo de Sófocles en su propia tragedia (Pearson 1917, II, p. 223, Radt 1999², p. 437, Sommerstein Fitzpatrick - Talboy 2006, pp. 146, 176), lo que explicaría la presencia de algunos rasgos de estilo sofocleo en S., Fr. 581 R. 
Arnott, W. G. 2007: Birds in the Ancient World from A to Z, London - New York.

Aubert, H. y Wimmer, Fr. 1868: Aristoteles Thierkunde Bd. I, Leipzig.

Biraud, M. y Delbey, E. 2006: «Philomèle: Du mythe aitiologique au début du mythe littéraire», Rursus 1, pp. 1-19 <http://rursus.revues.org/45> (04/03/2014).

Brown, M. K. 2002: The Narratives of Konon, München - Leipzig.

Burnett, A. P. 1998: Revenge in Attic and Later Tragedy, Berkeley - Los Angeles - London.

Casanova, A. 2006: «Filomela da rondine a usignolo», Concentus ex dissonis. Scritti in onore di Aldo Setaioli, Napoli 2006, vol. 1, pp. 165-178.

Cazzaniga, I. 1950: La saga di Itis nella tradizione letteraria e mitografica grecoromana.Parte prima: la tradizione letteraria e mitografica greco-romana da Omero a Nonno Panopolitano, Milano.

Chantraine, P. $1999^{2}$ : Dictionnaire étymologique de la langue grecque, Paris.

Coo, L. 2013: «A Tale of Two Sisters: Studies in Sophocles' Tereus», TAPhA 143.2, pp. 349-385.

Curley, D. 2003: «Ovid's Tereus: Theater and Metatheater», en Sommerstein, A. H. (ed.), Shards from Kolonos: Studies in Sophoclean Fragments, Bari, pp. 163-197.

Denniston, J. D. 1954²: The Greek Particles, Oxford.

Diggle, J. 1998: Tragicorum graecorum fragmenta selecta, Oxford.

Dobrov, G. 1993: «The tragic and the comic Tereus», AJPh 114, pp. 189-243.

Dubel, S. 2006: «L'hirondelle et l'épervier, le rossignol et la huppe (Achille Tatius, Leucippé et Clitophon, V, 3-5): notes sur la difficulté d'établir un mythe», en Gély, V. et al. (eds.), PHILOMELLE. Figures du rossignol dans la tradition littéraire et artistique, Clermont-Ferrand, pp. 37- 52.

Dunbar, N. 1995: Aristophanes. Birds, Oxford.

Escalígero, J. J. 1573: Publii Virgilii Maronis Appendix, cum supplemento multorum antehac nunquam excusorum poematum veterum poetarum; Josephi Scaligeri in eandem Appendicem commentarii et castigationes, Lugduni.

Fitzpatrick, D. 2001: «Sophocles’ Tereus», CQ 51.1, pp. 90-101.

Fontenrose, J. 1948. «The Sorrows of Ino and Procne», TAPhA 79, pp. 125-167.

Forbes Irving, P. M. C. 1990. Metamorphosis in Greek Myths, Oxford.

Friis Johansen, H. y Whittle, E. W. 1980: Aeschylus. The Suppliants vol. II, København.

Gildenhard, I. y Zissos, A. 2007: «Barbarian variations: Tereus, Procne and Philomela in Ovid (Met. 6.412-674) and Beyond», Dictynna 4, pp. 1-10.

Hanhemann, C. 2012: «Sophoclean fragments», en Ormand, K. (ed.), A Companion to Sophocles, Chichester, pp. 169-184.

Heath, B. 1762: Tragicorum graecorum veterum Aeschyli Sophoclis Euripidis quae supersunt dramata deperditorumque relliquiae, Oxonii.

Hiller, F. 1886 : De graecorum fabulis ad Thraces pertinentibus quaestiones criticae, Berolini. 
Hume, R. 2011: Guía de campo de las aves de Europa y España, Barcelona.

Kaufhold, S. D. 1997: «Ovid's Tereus: Fire, Birds, and the Reification of Figurative Language», CPh 92.1, pp. 66-71.

Keller, O. 1913: Die antike Tierwelt. Bd 2, Leipzig.

Kunstmann, J. G. 1938: The Hoopoe, a Study in European Folklore, Chicago.

Lloyd-Jones, H. 1996: Sophocles. Fragments, Cambridge (Mass.)-London.

March, J. 2000: «Vases and Tragic Drama: Euripides' Medea and Sophocles' Lost Tereus», en Rutter, N. K. y Sparkes, B. A. (eds.), Word and Image in Ancient Greece, Edinburgh, pp. 118-139.

March, J. 2003: «Sophocles' Tereus and Euripides' Medea», en Sommerstein, A. H. (ed.), Shards from Kolonos: Studies in Sophoclean Fragments, Bari, pp. 139-161.

Martín Rodríguez, A. Ma . 2002: De Aedón a Filomela. Génesis, sentido y comentario de la versión ovidiana del mito, Las Palmas de Gran Canaria.

Mette, H. J. 1959: Die Fragmente der Tragödien des Aischylos, Berlin.

Mette, H. J. 1963: Der verlorene Aischylos, Berlin.

Milo, D. 2008: Il Tereo di Sofocle, Napoli.

Monella, P. 2005: Procne e Filomela: dal mito al simbolo letterario, Bologna.

Nauck, A. 1889²: Tragicorum Graecorum Fragmenta, Lipsiae.

Neuhausen, K. A. 1969: «Tereus und die Danaiden bei Aischylos», Hermes 97, pp. 167-186.

Oder, E. 1888: «Der Wiedehopf in der griechischen Sage», RhM 43, pp. 541-556.

Pearson, A. C. 1917: The Fragments of Sophocles vol. II, Cambridge.

Peerlkamp, P. H. 1834: Q. Horatii Flacci Carmina, Harlemi.

Pollard, J. 1977: Birds in Greek Life and Myth, London.

Privitera, T. 2007: Terei puellae: metamorfosi latine, Pisa.

Radke, G. 1957: «Prokne», RE, coll. 247-252.

Radt, S. 19992: Tragicorum Graecorum fragmenta. Vol. 4: Sophocles, Göttingen.

Sandin, P. 2005: Aeschylus' Supplices. Introduction and Commentary on vv. 1-523, Lund.

Scattolin, P. 2013: «Le notizie sul Tereo di Sofocle nei papiri», en Bastianini, G. y Casanova, A. (eds.), I papiri di Eschilo e di Sofocle. Atti del convegno internazionale di studi, Firenze, 14-15 giugno 2012, Firenze, pp. 119-141.

Schroeder, O. 1926: «ПPOKNH», Hermes 61, pp. 423-436.

Sommerstein, A. H. 1991²: Aristophanes. Birds, Oxford.

Sommerstein, A. H., Fitzpatrick, D. y Talboy, T. 2006: Sophocles. Selected Fragmentary Plays I, Oxford.

Sutton, D. F. 1984: The Lost Sophocles, Lanham.

Svensson, L. 2010: Guía de aves de España, Europa y Región Mediterránea, Madrid. Thompson, D. W. 1895: A Glossary of Greek Birds, Oxford.

Van Leeuwen, J. 1902: Aristophanis Aves, Lugduni Batavorum. 
Wecklein, N. 1893: Aeschyli fabulae, cum lectionibus et scholiis codicis Medicei et in Agamemnonem codicis Florentini ab Hieronymo Vitelli denuo collatis vol. I: fragmenta, Berolini.

Welbergen, J. A. y Davies, N. B. 2011: «A Parasite in Wolf's Clothing: Hawk Mimicry Reduces Mobbing of Cuckoos by Hosts», Behavioral Ecology 22.3, pp. 574-579.

Welcker, G. 1839: Die griechischen Tragödien mit Rücksicht auf den epischen Zyklus geordnet, I, Bonn.

Wright, M. 2012: «The Reception of Sophocles in Antiquity», en A. Markantonatos (ed.), Brill's Companion to Sophocles, Leiden - Boston, pp. 581-599.

Fecha de recepción de la primera versión del artículo: 20/03/2014

Fecha de aceptación: 08/07/2014

Fecha de recepción de la versión definitiva: 28/10/2014 